\title{
Regeneración de un bosque mixto de Nothofagus (Nothofagaceae) después de una corta selectiva
}

\author{
Regeneration of a Nothofagus (Nothofagaceae) mixed forest after selective cutting \\ ALEJANDRO DEZZOTTI, RENATO SBRANCIA, MARCELO RODRÍGUEZ-ARIAS, \\ DANIELA ROAT \& ANDREA PARISI
}

\author{
Asentamiento Universitario San Martín de los Andes, Universidad Nacional del Comahue, \\ Pasaje de la Paz 235, Q8370AQA San Martín de los Andes, Argentina; \\ e-mail: dezzotti@infovia.com.ar
}

\begin{abstract}
RESUMEN
El estudio de la ecología de la regeneración natural del bosque constituye una parte esencial de la silvicultura. El cerro Tren Tren $\left(40^{\circ} 12^{\prime} \mathrm{S}, 7^{\circ} 26^{\prime} \mathrm{O}, 1.367 \mathrm{~m}\right.$, Reserva Mapuche Curruhuinca, Argentina) está ocupado por un bosque cerrado compuesto por Nothofagus dombeyi, N. nervosa y N. obliqua. En 1994 se estimó la densidad de renovales (diámetro en la base del tallo $<10 \mathrm{~cm}$ ) en 90 ind $\mathrm{ha}^{-1}$. Posteriormente, se implementaron cortas de selección en bosquetes y los 10 claros artificiales resultantes de entre 1.587 y 4.322 $\mathrm{m}^{2}$ se clausuraron al ganado. En 2002 las estructuras de tamaño y edad de los renovales se estimaron a través de muestreos sistemáticos y aleatorios. Las tres especies de Nothofagus se establecieron en forma simultánea en los claros artificiales. Sin embargo, la especie más tolerante a la sombra $N$. nervosa exhibió una edad media ligeramente mayor debido a su persistencia como "regeneración avanzada" antes de las cortas. La densidad de renovales se estimó en 97.006 ind $\mathrm{ha}^{-1}$ (68\% N. dombeyi, $20 \% \mathrm{~N}$. obliqua y $12 \% \mathrm{~N}$. nervosa). Los renovales mostraron una abundancia y composición independiente de la forma y tamaño de los claros, y de la abundancia relativa de árboles adultos que conformaban el rodal y el límite de los claros. En la zona clausurada los renovales ramoneados representaron el $15 \%$ mientras que en la zona no clausurada el $33 \%$, una diferencia atribuible al efecto protector de la clausura. El $86 \%$ de los renovales se localizaron en las áreas con niveles bajos e intermedios de altura y cobertura del sotobosque. El plan de manejo se considera adecuado si se compara el proceso de regeneración del bosque antes y después de su implementación, y se abren perspectivas alentadoras para la conservación de la comunidad dominada por Nothofagus bajo este sistema silvicultural.
\end{abstract}

Palabras clave: Nothofagus dombeyi, Nothofagus obliqua, Nothofagus nervosa, manejo forestal, Norpatagonia.

\begin{abstract}
Understanding forest regeneration is essential to develop sound, ecologically-based silvicultural practices. Mount Tren Tren $\left(40^{\circ} 12^{\prime}\right.$ S, $71^{\circ} 26^{\prime}$ W, 1,367 m, Reservation Mapuche Curruhuinca, Argentina) is occupied by a closed stand of Nothofagus dombeyi, N. obliqua, and N. nervosa. In 1994, tree regeneration (root collar diameter $<10 \mathrm{~cm}$ ) was estimated to be 90 ind $\mathrm{ha}^{-1}$. After that, a group selection silvicultural system was implemented and the resulting 10 artificial gaps of between 1,587 and $4,322 \mathrm{~m}^{2}$ were enclosed to prevent grazing by domestic livestock. Size and age structure of regeneration were analysed based on systematic and random samplings. All tree species became established simultaneously within the artificial gaps. However, the less light demanding $N$. nervosa exhibited a mean age slightly larger given its differential persistence as "advanced regeneration" previous to cutting treatment. Tree regeneration was estimated in 97,006 ind ha ${ }^{-1}$ (68\% of N. dombeyi, $20 \%$ of $N$. obliqua, and $12 \%$ of $N$. nervosa). Regeneration abundance and composition were unrelated to shape and size of regeneration gaps, nor to abundance of adult trees within the stand and along the gap boundaries. A significant positive effect of the enclosure on plant was observed. The $86 \%$ of juvenile Nothofagus were present when understory height and cover were low to intermediate. The management plan is considered adequate if the abundance of regeneration is compared before and after its implementation, and encourages the use and conservation of this forest type under the current silvicultural system.
\end{abstract}

Key words: Nothofagus dombeyi, Nothofagus obliqua, Nothofagus nervosa, forest management, northern Patagonia. 


\section{INTRODUCCIÓN}

La presión del hombre sobre los bosques nativos para obtener productos tangibles o áreas para la ganadería, agricultura y urbanización promueve la simplificación, desaparición y degradación de estos ecosistemas (Goudie 1993, Morello \& Matteucci 1999). El manejo forestal se debe llevar a cabo desde una perspectiva de sustentabilidad ecológica y para ello la silvicultura debe imitar al régimen de las perturbaciones naturales y ser compatible con las estrategias de regeneración de las plantas (Aplet et al. 1993, Smith et al. 1997). Aunque los renovales constituyen la fase temprana y más frágil del ciclo de vida del árbol, contribuyen a mantener la continuidad del bosque en el tiempo y en el espacio, y consecuentemente representan una indicadora de manejo conservativo (Wagner \& Zasada 1991, Raison et al. 2001).

El género Nothofagus ${ }^{1}$ está conformado por árboles diclino monoicos que exhiben dispersión anemócora, dependencia en la reproducción sexual para la colonización de nuevos hábitats y ciclos reproductivos de cuatro años consecutivos para la formación de una cohorte de propágulas (Poole 1987). Sus especies sudamericanas se comportan como plantas sucesionales tempranas que recolonizan sitios abiertos luego de la eliminación de la vegetación original a causa de la presencia de disturbios periódicos y de gran escala (Veblen 1989). En ausencia de tales perturbaciones, plantas sucesionales tardías tienden a establecerse en forma continua bajo el dosel y a reemplazar sucesionalmente a las especies de Nothofagus que no toleran condiciones de escasa luminosidad. Sin embargo, la gran intensidad y alta frecuencia, relativa a la longevidad de Nothofagus, de los fenómenos catastróficos masivos asociados a las áreas de montaña retrasan el proceso de exclusión competitiva y proveen las condiciones para el nuevo establecimiento de Nothofagus (Veblen et al. 1981). En la Argentina, las especies de Nothofagus no son reemplazadas sucesionalmente debido a la ausencia de especies arbóreas tolerantes a la sombra (Veblen 1989).

Los bosques compuestos por $N$. dombeyi (coihue), $N$. obliqua (roble pellín) y $N$. nervosa $(\text { raulí })^{2}$ se distribuyen sobre las laderas húmedas de las cordilleras de la Costa y los Andes, aproximadamente entre los $39^{\circ} 29^{\prime}$ y $40^{\circ} 22^{\prime} \mathrm{S}$ y

\footnotetext{
${ }^{1}$ Existe consenso para incluir a Nothofagus en la familia monogenérica Nothofagaceae (Hill \& Jordan 1993).

${ }^{2}$ La nomenclatura del raulí se basa en Lennon et al. (1987) y la de las demás especies en Correa $(1978,1984)$.
}

hasta los $1.000 \mathrm{~m}$ (Lara et al. 1999). Estudios experimentales, ecofisiológicos y poblacionales indican la existencia de divergencias interespecíficas con relación a la sobrevivencia, el crecimiento y desarrollo de los renovales bajo diferentes regímenes de luminosidad (Alberdi 1976, Read \& Hill 1985, Grosse 1988b, Ramírez et al. 1997, Weinberger \& Ramírez 2001). La silvicultura de esta comunidad se basa primariamente en cortas sucesivas de protección que producen un rodal coetáneo (Grosse \& Quiróz 1998, Lara et al. 1998, Martínez-Velásquez 1998). Sin embargo, Nothofagus es susceptible también de manejarse a través de cortas de selección en bosquetes que originan un rodal multietáneo (Chauchard et al. 1994³ , MartínezVelásquez 1998). Este sistema consiste en talar los árboles de mayor tamaño de un rodal a intervalos repetidos denominados ciclos de corta. La superficie total de corta se distribuye en claros artificiales donde se proveen las condiciones de luminosidad que favorecerían el establecimiento de los renovales, mientras que los árboles circundantes proporcionarían su protección contra la deshidratación y el congelamiento (Smith et al. 1997).

El objetivo de este estudio es evaluar los efectos de las cortas de selección en bosquetes después de ocho años de su implementación, sobre la abundancia, composición y estado de conservación de renovales de $N$. dombeyi, $N$. obliqua y $N$. nervosa que se encuentran protegidos contra la herbivoría de animales domésticos a través de clausuras de los claros artificiales. La densidad actual de renovales se compara con la existente antes de la corta de selección. Se espera que las aperturas del dosel hayan provocado cambios en el régimen de luz y en los parámetros microclimáticos dependientes, promoviendo la regeneración de especies arbóreas resguardadas del pastoreo del ganado.

\section{MATERIALES Y MÉTODOS}

El área de estudio se localiza sobre las laderas $\mathrm{S}$ y O del cerro Tren Tren $\left(40^{\circ} 12^{\prime}\right.$ S, $71^{\circ} 26^{\prime} \mathrm{W}, 1.367$ m, Parque Nacional Lanín, Argentina) (Fig. 1). Se caracteriza por un clima templado húmedo con una estación estival seca contrastante. La temperatura media y la precipitación anual se estiman en $8,8{ }^{\circ} \mathrm{C}$ y $2.360 \mathrm{~mm}$, respectivamente, y los

\footnotetext{
3 CHAUCHARD L, A FORGUES \& M FERNÁNDEZ (1994) Plan de manejo cerro Tren Tren. Asentamiento Universitario San Martín de los Andes, Universidad Nacional del Comahue, San Martín de los Andes, Argentina. Manuscrito, $21 \mathrm{pp}$.
} 


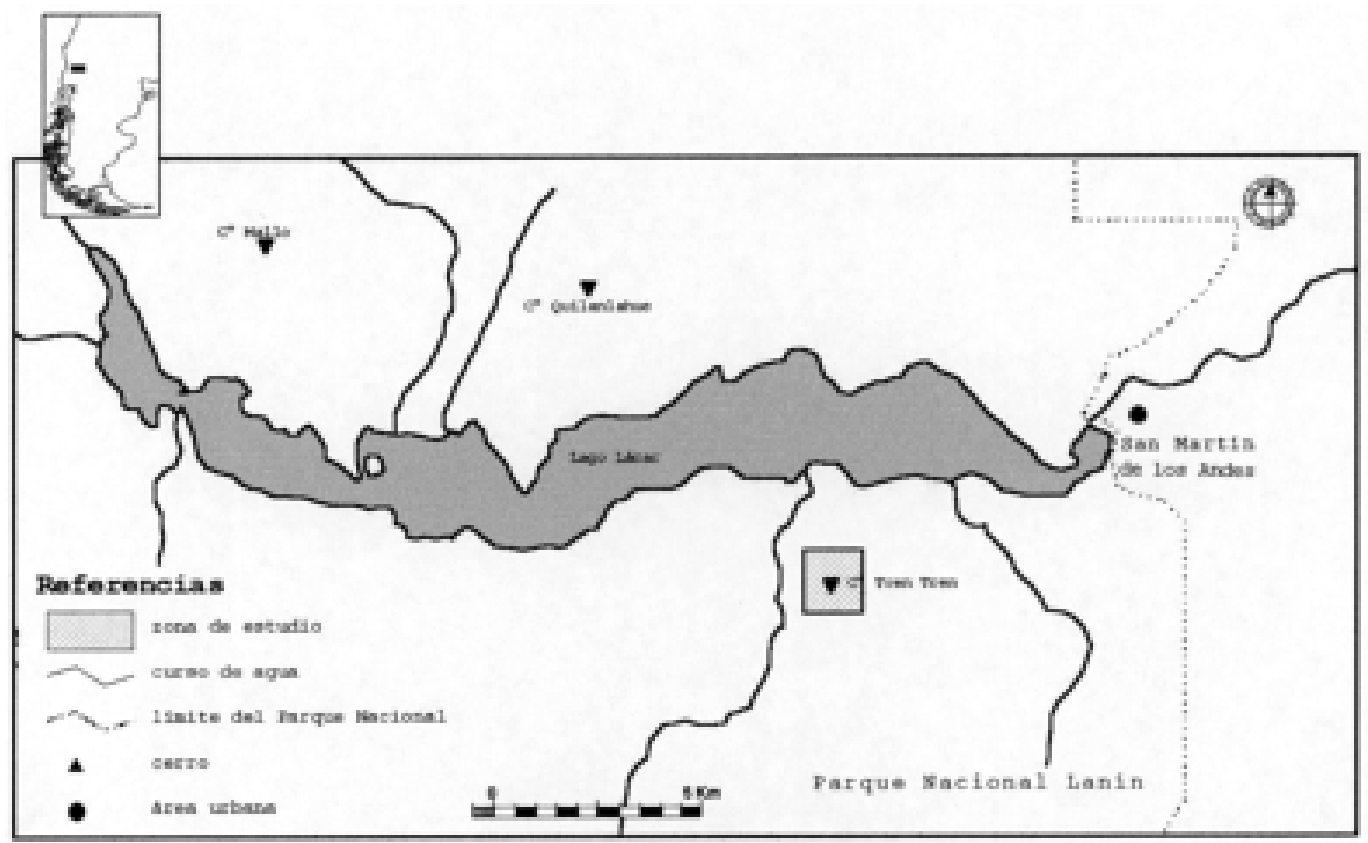

Fig. 1: Mapa del área de estudio localizada entre los $40^{\circ} 06^{\prime}$ y $40^{\circ} 12^{\prime} \mathrm{S}$ y entre los $71^{\circ} 19^{\prime}$ y $71^{\circ} 40^{\prime} \mathrm{O}$. Map of the study area located between $40^{\circ} 06^{\prime}$ and $40^{\circ} 12^{\prime} \mathrm{S}$, and between $71^{\circ} 19^{\prime}$ and $71^{\circ} 40^{\prime} \mathrm{W}$.

vientos generalmente provienen del O y NO. El relieve es montañoso con pendientes máximas mayores a $30 \%$ y moderada variación de las elevaciones. Los suelos dominantes se clasifican como Andosoles, y se caracterizan por la alta capacidad de retención de agua y de fosfatos, de permeabilidad a los fluidos y de intercambio catiónico, por el alto contenido de limo, arcilla y materia orgánica y por el $\mathrm{pH}$ levemente ácido (Ferrer et al. 1990). Fitogeográficamente, la vegetación pertenece al Distrito del Bosque Caducifolio dentro de la Provincia Subantártica (Cabrera 1971). El área de estudio está ocupada por pobladores rurales de la Comunidad Mapuche Curruhuinca que se dedican principalmente a la ganadería de bovinos, ovinos y equinos y a la extracción de Chusquea culeou (caña colihue, Gramineae), madera y leña. En 1994 se implementó un aprovechamiento forestal basado en el sistema silvicultural de cortas de selección en bosquetes y se formaron diez claros artificiales (Fig. 2), a los que se les colocaron alambrados perimetrales que delimitaron zonas clausuradas y no clausuradas al ganado.

En el bosque predominan $N$. dombeyi, $N$. obliqua y $N$. nervosa, aunque también se encuentran individuos aislados de $N$. antarctica (ñire), N. pumilio (lenga), Austrocedrus chilensis (ciprés de la cordillera, Cupressaceae) y Maytenus boaria (maitén, Celastraceae). En el estrato arbustivo se encuentran principalmente
C. culeou, Berberis darwinii (michay, Berberidaceae) y Azara microphylla (chin-chin, Flacourtiaceae), y en el herbáceo Acaena ovalipholia (cadillo, Rosaceae), Osmorrhiza chilensis (cacho de cabra, Umbeliferae) y Alstroemeria aurea (amancay, Amarilidaceae). Chauchard et al. (1994) ${ }^{3}$ estimaron el tamaño del rodal en 9,6 ha, la densidad en 206 ind ha ${ }^{-1}$ (45\% de N. dombeyi, $16 \%$ de N. obliqua y 39 $\%$ de $N$. nervosa), el área basal en $55,8 \mathrm{~m}^{2} \mathrm{ha}^{-1}$ (43, 20 y $37 \%$ de cada especie de Nothofagus, respectivamente) y la altura media de los árboles dominantes en $32,3 \mathrm{~m}$. El rodal presentó una distribución diamétrica levemente asimétrica hacia la derecha (Chauchard et al. 1994) ${ }^{3}$, indicativa de la coetaneidad de las poblaciones en razón de la relación frecuentemente observada entre la edad y el diámetro de los árboles individuales de especies heliófilas. En el rodal se extrajeron $850 \mathrm{~m}^{3}$ de rollizos $(40 \%$ de $N$. dombeyi, $12 \%$ de $N$. obliqua y $48 \%$ de $N$. nervosa), los cuales se retiraron hasta los caminos principales con bueyes (Monópoli 1997) ${ }^{4}$. La densidad de renovales (diámetro en la base del tallo DB $<10 \mathrm{~cm}$ ) previa a la implementa-

\footnotetext{
${ }^{4}$ MONOPOLI J (1997) Evaluación y seguimiento de un plan de manejo forestal. Asentamiento Universitario San Martín de los Andes, Universidad Nacional del Comahue, San Martín de los Andes, Argentina. Manuscrito, 27 pp.
} 


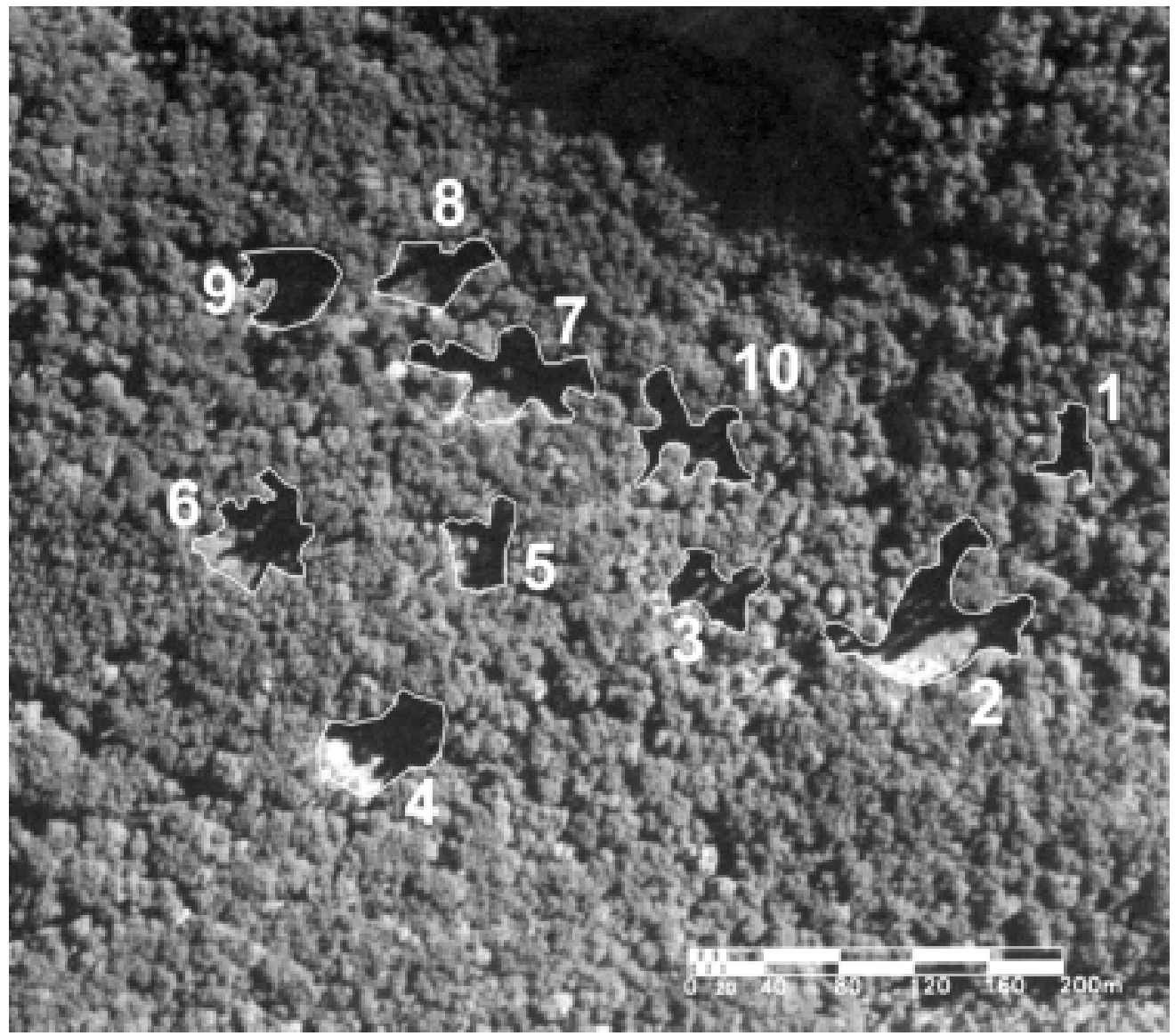

Fig. 2: Fotografía aérea del cerro Tren Tren indicando la localización de los 10 claros artificiales (tomada en abril de 2001 , escala $\approx 1: 3.000$ ). Las características de los claros se indican en la Tabla 1.

Aerial photo of Mount Tren Tren showing the location of the 10 artificial gaps (taken in April 2001, scale $\approx 1: 3,000$ ). Gap characteristics are indicated in Table 1.

ción del plan de manejo se estimó en 90 ind ha-1 (Chauchard et al. 1994) ${ }^{3}$.

Para establecer la arquitectura de los claros, a cada Nothofagus adulto vivo (diámetro a la altura del pecho DAP, $1,3 \mathrm{~m}$ del suelo, $\geq 10$ $\mathrm{cm})$ que conformaba el límite de los claros se lo numeró y se le midió el DAP (cinta diamétrica) y la proyección perpendicular de la copa (cinta métrica). Luego se midieron la distancia (cinta métrica), el acimut (brújula) y la pendiente (clinómetro) entre estos árboles y entre los vértices del alambrado que delimitaba la zona clausurada. Mediante el programa AUTOCAD se estimaron las áreas expandidas y efectivas de los claros, delimitadas por los troncos y las copas de los árboles que los circunscribían, respectivamente (sensu Runkle 1992), y las áreas excluidas al ganado, definidas por la presencia del alambrado. Se midieron la exposición y pendiente máxima de cada claro, y se calculó su índice de forma que re- presentó el cociente entre el perímetro del claro expandido y el perímetro de un círculo con una área equivalente.

Las estructuras de tamaño y edad de los renovales se estimaron a través de muestreos sistemáticos y aleatorios. Al plano de cada claro se le superpuso una grilla cuyas intersecciones correspondían a la posición de 511 parcelas cuadradas de $0,5 \mathrm{~m}^{2}$ cada una. La equidistancia entre las transectas varió para garantizar la inclusión de al menos 20 parcelas de muestreo dentro de la zona clausurada de cada claro. En cada parcela se contaron los renovales de acuerdo a la especie, la posición (zona clausurada y no clausurada), los estados de desarrollo plantín (altura total AT $<30 \mathrm{~cm}$ ) y juvenil $(\mathrm{AT} \geq 30 \mathrm{~cm})$ y los estados de conservación sano, enfermo (moribundo o afectado por algún agente patógeno) y comido (presencia de lesiones producidas por herbívoros vertebrados). En cada parcela se estableció la cobertura del sotobosque (CS) que representó el 
porcentaje de la unidad de muestreo ocupada por la proyección perpendicular de las partes aéreas de las especies del sotobosque, de acuerdo a las categorías 0 (sotobosque ausente), $1(\leq 25 \%), 2$ $(25-50 \%)$ y $3(\geq 50 \%)$, y la altura media del sotobosque (AS) de acuerdo a las categorías 1 $(\mathrm{AT}<30 \mathrm{~cm}), 2(30 \leq \mathrm{AT}<130 \mathrm{~cm})$, y $3(\mathrm{AT} \geq$ $130 \mathrm{~cm})$. Para estimar el efecto del sotobosque sobre el desarrollo de los renovales se creó la variable semicuantitativa dominancia del sotobosque (DS) que correspondió al producto de CS y AS. Se consideró que la influencia de la cobertura del sotobosque sobre el desarrollo de los renovales de Nothofagus era equivalente a la de la altura del sotobosque. Para ello, DS $=0=$ dominancia nula, DS = 1 ó $2=$ dominancia baja, $\mathrm{DS}=3$ ó $4=$ dominancia intermedia y $\mathrm{DS}=6$ ó $9=$ dominancia alta. La edad de los renovales se estimó bajo lupa, contando las cicatrices dejadas por las escamas de las yemas anuales en 1.133 individuos recolectados al azar de los claros 2, 4, 5 y 6. La normalidad de los datos y la homogeneidad de varianzas se analizaron a través de las pruebas de chi-cuadrado y de Bartlett $(\mathrm{P}<0,05)$, respectivamente (Sokal \& Rohlf 1995). Los análisis estadísticos se realizaron a través del programa STATGRAPHICS.

\section{RESULTADOS}

La superficie de los claros expandidos varió entre 1.587 y $4.322 \mathrm{~m}^{2}$, y la de los claros efectivos entre 766 y $3.870 \mathrm{~m}^{2}$. Los claros 1 y 2 exhibieron el menor y el mayor tamaño de claro expandido, efectivo y de la clausura, respectivamente (Tabla 1). El DAP medio de los árboles adultos que conformaron el límite de cada claro varió entre 63 (claro 4) y $95 \mathrm{~cm}$ (claro 1). Los árboles de menor y mayor tamaño exhibieron un DAP de 22 (claro 10, N. nervosa) y 181 $\mathrm{cm}$ (claro 10, $N$. dombeyi), respectivamente. Nothofagus nervosa presentó su mayor densidad en el borde de los claros 2 (54\% del total de árboles), 3 (62\%), 4 (67\%), 5 (45\%), 7 (69\%), $8(52 \%)$ y $10(59 \%)$, mientras que $N$. dombeyi en los claros $1(63 \%)$ y $9(75 \%)$, y $N$. obliqua en el claro $6(61 \%)$. $N$. obliqua se encontró ausente en los claros 2 y 10 (Tabla 2).

Las edades medias de los renovales de $N$. dombeyi, $N$. obliqua y $N$. nervosa se estimaron en 3,8, 4,0 y 4,3 años, respectivamente. Las edades mínima y máxima de los individuos se estimaron en 2 (para todas las especies) y 10 años (para $N$. nervosa), respectivamente. La edad media de $N$. nervosa difirió estadísticamente de las

TABLA 1

Características físicas de los 10 claros artificiales y volumen bruto de madera rolliza obtenido durante la aplicación del tratamiento silvícola en 1994. Se indican la pendiente máxima (P) y exposición (E) de los claros. Nd: $N$. dombeyi, No: $N$. obliqua y $N n: N$. nervosa

Physical characteristics of the 10 artificial gaps and gross timber volume obtained during the implementation of the silvicultural system in 1994. Maximum slope (P) and topographic direction (E) of gaps are shown. Nd: N. dombeyi, No: N. obliqua, and Nn: N. nervosa

\begin{tabular}{|c|c|c|c|c|c|c|c|c|c|}
\hline \multirow[t]{3}{*}{ Claro } & \multirow[t]{3}{*}{$\mathrm{P}\left({ }^{\circ}\right)$} & \multirow[t]{3}{*}{$\mathrm{E}$} & \multicolumn{3}{|c|}{ Área $\left(\mathrm{m}^{2}\right)$} & \multicolumn{4}{|c|}{ Volumen $\left(\mathrm{m}^{3}\right)$} \\
\hline & & & \multirow[t]{2}{*}{ Clausura } & \multicolumn{2}{|c|}{ Claro } & \multirow[t]{2}{*}{$N d$} & \multirow[t]{2}{*}{ No } & \multirow[t]{2}{*}{$N n$} & \multirow[t]{2}{*}{ Total } \\
\hline & & & & Expandido & Efectivo & & & & \\
\hline 1 & 8 & SSO & 577 & 1.587 & 766 & 13,4 & 0 & 13,5 & 26,9 \\
\hline 2 & 9 & SSE & 2.563 & 4.322 & 3.870 & 96,0 & 0 & 42,8 & 138,7 \\
\hline 3 & 7 & SSE & 1.385 & 2.195 & 1.321 & 20,3 & 3,7 & 40,3 & 64,3 \\
\hline 4 & 33 & OSO & 1.344 & 3.373 & 2.433 & 0 & 11,2 & 86,3 & 97,5 \\
\hline 5 & 6 & SE & 1.466 & 2.022 & 1.258 & 50,7 & 10,7 & 12,9 & 74,3 \\
\hline 6 & 10 & SSE & 2.140 & 2.747 & 2.260 & 9,7 & 72,8 & 20,5 & 103,0 \\
\hline 7 & 16 & ENE & 1.200 & 2.968 & 2.724 & 0 & 0,4 & 105,2 & 105,6 \\
\hline 8 & 16 & SSE & 1.178 & 2.125 & 1.462 & 42,1 & 9,0 & 40,9 & 92,0 \\
\hline 9 & 34 & SSE & 986 & 2.175 & 1.579 & 72,5 & 0 & 15,7 & 88,2 \\
\hline \multirow[t]{2}{*}{10} & 8 & SSO & 1.123 & 3.090 & 1.804 & 33,4 & 0 & 25,3 & 58,8 \\
\hline & Total & 13.962 & 26.604 & 19.477 & & 338,0 & 107,8 & 403,3 & 849,1 \\
\hline
\end{tabular}


TABLA 2

Densidad de árboles adultos que conforman el límite de cada claro y de los renovales presentes en los claros artificiales en función de las especies y los estados de desarrollo.

Se indican la media, el error estándar (EE) y el tamaño de la muestra (n).

Nd: N. dombeyi, No: N. obliqua y Nn: N. nervosa

Density of adult trees that circumscribe the gaps and regeneration within the artificial gaps by species and developmental stages. Mean, standard error (EE), and sample size (n) are shown $N d$ : N. dombeyi, No: N. obliqua, and Nn: N. nervosa

\begin{tabular}{|c|c|c|c|c|c|c|c|c|c|c|c|}
\hline \multirow{2}{*}{ Claro } & \multicolumn{4}{|c|}{ Adultos } & \multicolumn{7}{|c|}{ Renovales } \\
\hline & $\mathrm{n}$ & $\begin{array}{l}N d \\
(\%)\end{array}$ & $\begin{array}{l}N o \\
(\%)\end{array}$ & $\begin{array}{l}\mathrm{Nn} \\
(\%)\end{array}$ & $\mathrm{n}$ & $\begin{array}{l}N d \\
(\%)\end{array}$ & $\begin{array}{l}\text { No } \\
(\%)\end{array}$ & $\begin{array}{l}\mathrm{Nn} \\
(\%)\end{array}$ & $\begin{array}{c}\text { Plantín } \\
(\%)\end{array}$ & $\begin{array}{c}\text { Juvenil } \\
(\%)\end{array}$ & $\begin{array}{c}\text { Total } \\
\left(\text { ind ha }{ }^{-1}\right)\end{array}$ \\
\hline 1 & 19 & 31,6 & 5,3 & 63,1 & 99 & 93,5 & 0 & 6,5 & 99,0 & 1,0 & 38.269 \\
\hline 2 & 35 & 54,3 & 0 & 45,7 & 396 & 91,2 & 3,2 & 5,6 & 91,2 & 8,8 & 141.429 \\
\hline 3 & 31 & 61,9 & 14,3 & 23,8 & 224 & 57,3 & 26,4 & 16,3 & 98,9 & 1,1 & 72.097 \\
\hline 4 & 34 & 66,7 & 12,1 & 21,2 & 99 & 66,7 & 23,2 & 10,1 & 92,9 & 7,1 & 36.667 \\
\hline 5 & 29 & 44,5 & 11,1 & 44,4 & 187 & 77,5 & 3,8 & 18,7 & 99,5 & 0,5 & 110.000 \\
\hline 6 & 38 & 16,7 & 61,1 & 22,2 & 738 & 55,6 & 40,7 & 3,7 & 92,8 & 7,2 & 301.224 \\
\hline 7 & 40 & 68,4 & 5,3 & 26,3 & 105 & 47,6 & 18,1 & 34,3 & 89,5 & 10,5 & 32.813 \\
\hline 8 & 34 & 51,5 & 3,0 & 45,5 & 258 & 76,7 & 7,8 & 15,5 & 100 & 0 & 109.787 \\
\hline 9 & 29 & 21,4 & 3,6 & 75,0 & 138 & 88,7 & 6,2 & 5,1 & 86,9 & 13,1 & 59.783 \\
\hline 10 & 34 & 59,4 & 0 & 40,6 & 235 & 47,7 & 20,9 & 31,4 & 70,6 & 29,4 & 100.000 \\
\hline Media & & 48,3 & 12,3 & 39,4 & & 68,0 & 20,1 & 11,9 & 92,0 & 8,0 & 97.006 \\
\hline $\mathrm{EE}$ & & 5,9 & 5,7 & 5,7 & & 5,6 & 4,1 & 3,4 & 2,8 & 2,8 & 25.196 \\
\hline
\end{tabular}

de $N$. obliqua y $N$. dombeyi, mientras que entre estas últimas especies no se observaron diferencias significativas (Tabla 3). Aunque las tres especies presentaron una distribución de edades que se alejó de la normal (prueba de Chi-cuadrado; $\mathrm{P} \leq 0,0001), N$. nervosa exhibió una distribución más asimétrica hacia la derecha (índice de simetría $\mathrm{z}=6,51 ; \mathrm{P}<0,0001)$ con respecto a $N$. dombeyi $(\mathrm{z}=3,29 ; \mathrm{P}=0,0011)$ y a $N$. obliqua $(\mathrm{z}$ $=3,33 ; \mathrm{P}=0,0009)$ (Fig. 3). Los juveniles de $N$. dombeyi y $N$. obliqua presentaron edades medias mayores que los plantines, sin embargo estos valores no difirieron estadísticamente. En cambio, para $N$. nervosa las edades de plantines y juveniles difirieron significativamente solo en 0,8 años (Tabla 3).

La densidad media de renovales se estimó en 97.006 ind ha $^{-1}$ (rango: $32.813-301.224$ ind $\mathrm{ha}^{-1}$ ) (Tabla 2). Nothofagus dombeyi fue la especie más abundante en todos los claros, representando 65.949 ind $\mathrm{ha}^{-1}$ (3,30 ind $0,5 \mathrm{~m}^{-2}$; EE $=0,50 ; 68 \%$ de la abundancia media), mientras que $N$. obliqua y $N$. nervosa representaron $19.550\left(0,98\right.$ ind $0,5 \mathrm{~m}^{-2} ; \mathrm{EE}=0,17 ; 20 \%$ de la abundancia media) y 11.507 ind $\mathrm{ha}^{-1}(0,59$ ind $0,5 \mathrm{~m}^{-2} ; \mathrm{EE}=0,11 ; 12 \%$ de la abundancia me- dia), respectivamente (prueba de Kruskal-Wa1lis; H3 = 42,448; $\mathrm{P}<0,0001 ; \mathrm{n}=511$ ) (Tabla 2). La densidad relativa de $N$. dombeyi varió entre $48 \%$ (claro 7) y $94 \%$ del total de renovales (claro 1), la de $N$. nervosa entre $4 \%$ (claro 6) y $34 \%$ (claro 7) y la de N. obliqua entre $0 \%$ (claro 1) y $41 \%$ (claro 6) (Tabla 2). Estas abundancias relativas no guardaron relación con la de los árboles adultos que constituyeron el rodal ni con la de adultos que conformaron los límites de los claros. Por ejemplo, N. nervo$s a$ representó el $38 \%$ de la densidad y el área basal del rodal y el $48 \%$ de los individuos que circunscriben los claros y solo aportó el $12 \%$ de los renovales (Tabla 2).

El $65 \%$ de los renovales se encontraron en la zona clausurada de los claros con una densidad media de 129.317 ind ha ${ }^{-1}\left(6,5\right.$ ind $0,5 \mathrm{~m}^{-2}$; $\mathrm{EE}=1,1 ; \mathrm{n}=249)$ y el $35 \%$ restante se hallaron en la zona no clausurada con 66.298 ind $\mathrm{ha}^{-1}\left(3,3\right.$ ind $\left.0,5 \mathrm{~m}^{-2} ; \mathrm{EE}=0,5 ; \mathrm{n}=262\right)($ Tabla 4). Estos valores difirieron significativamente entre sí (prueba de Kruskal-Wallis; H2 = 4,073; $\mathrm{P}=0,044)$. La densidad de renovales de las parcelas de las zonas clausurada y no clausurada adyacentes al alambrado se estimaron en 1,9 


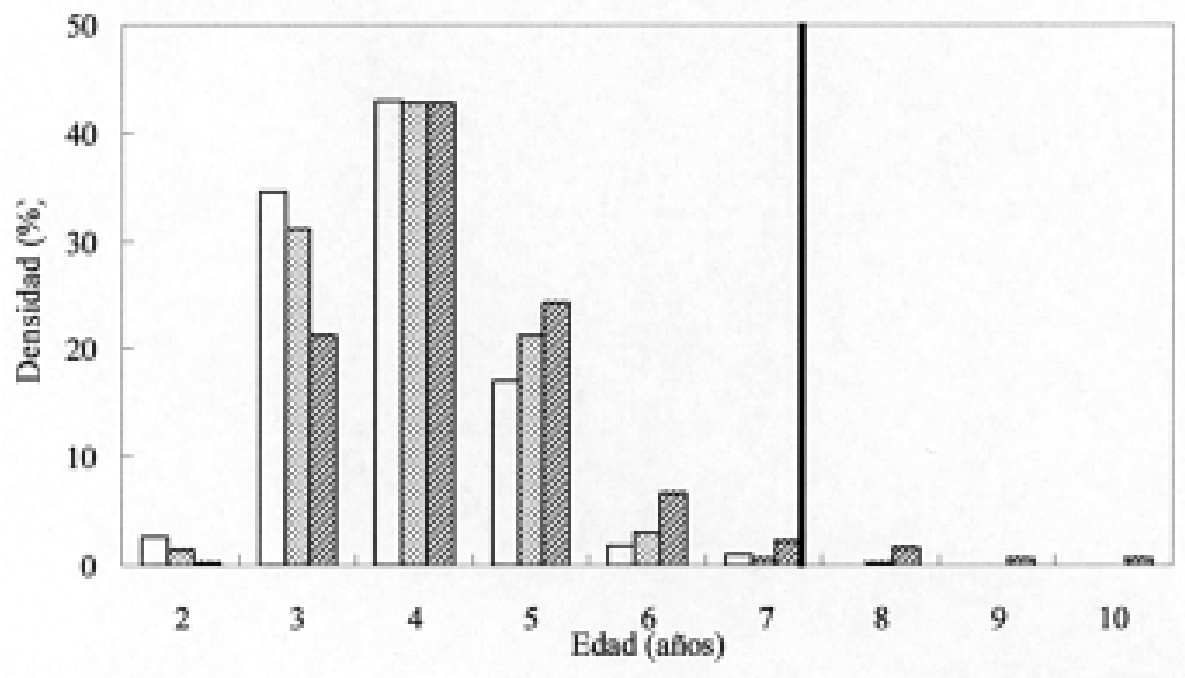

Fig. 3: Distribución de edades de los renovales de N. dombeyi (barras blancas), N. obliqua (barras de puntos) y $N$. nervosa (barras rayadas) en los claros artificiales. La línea vertical indica el año de la corta.

Age frequency distribution of $N$. dombeyi (open bars), $N$. obliqua (dotted bars), and $N$. nervosa (shaded bars) regeneration within the artificial gaps. Vertical line indicates the year when logging occurred.

TABLA 3

Edad (años) en función del estado de desarrollo de los renovales de Nothofagus.

Se indican la media, la mínima (mín), la máxima (máx), el error estándar (EE), el tamaño de la muestra (n) y los grupos homogéneos (GH). Letras minúsculas y mayúsculas desiguales indican diferencias significativas de medias entre estados de desarrollo y entre especies, respectivamente (prueba de Kruskal-Wallis; $\mathrm{P}<0,0001$ )

Age (yr) in relation to development stages of Nothofagus regeneration. Mean, minimum (mín), maximum (máx), standard error (EE), sample size (n), and homogeneous groups $(\mathrm{GH})$ are showed. Different small and capital letters indicate significant differences among stages and species, respectively (Kruskal-Wallis test, $\mathrm{P}<0.0001$ )

\begin{tabular}{|c|c|c|c|c|c|c|c|c|c|}
\hline \multirow[t]{2}{*}{ Estadístico } & \multicolumn{3}{|c|}{ N. dombeyi } & \multicolumn{3}{|c|}{ N. obliqua } & \multicolumn{3}{|c|}{ N. nervosa } \\
\hline & Plantín & Juvenil & Total & Plantín & Juvenil & Total & Plantín & Juvenil & Total \\
\hline Media & 3,84 & 3,94 & 3,84 & 3,91 & 4,04 & 3,96 & 4,01 & 4,90 & 4,34 \\
\hline Mín & 2 & 2 & 2 & 2 & 2 & 2 & 2 & 3 & 2 \\
\hline Máx & 5 & 7 & 7 & 6 & 8 & 8 & 7 & 10 & 10 \\
\hline $\mathrm{EE}$ & 0,05 & 0,09 & 0,05 & 0,05 & 0,09 & 0,05 & 0,05 & 0,12 & 0,06 \\
\hline GH & $\mathrm{a}$ & $\mathrm{a}$ & A & $\mathrm{a}$ & $\mathrm{a}$ & A & $\mathrm{a}$ & $\mathrm{b}$ & B \\
\hline $\mathrm{n}$ & 240 & 140 & 380 & 237 & 140 & 377 & 236 & 140 & 376 \\
\hline
\end{tabular}

$* 10^{6}(\mathrm{n}=129)$ y $3,8 * 10^{6}$ ind $\mathrm{ha}^{-1}(\mathrm{n}=140)$, respectivamente. La abundancia de renovales no guardó ninguna relación con el área (Análisis de varianza; $\left.\mathrm{F}_{1,8}=0,29 ; \mathrm{P}=0,607\right)$ y el índice de forma de los claros (Análisis de varianza; $\mathrm{F}_{1.8}=0,38 ; \mathrm{P}=0,553$ ) (Fig. 4). Por un lado, el claro 6 presentó un tamaño expandido de $2.747 \mathrm{~m}^{2}$ (el más cercano al promedio) y una densidad de 301.224 ind ha-1 (el más denso), mientras que el claro 2 presentó un tamaño expandido de $4.322 \mathrm{~m}^{2}$ (el más grande) y una densidad de 141.429 ind ha $^{-1}$.

En la zona clausurada el $75 \%$ de los renovales se encontraban sanos, el $15 \%$ ramoneados y el $10 \%$ enfermos, mientras que en la zona no clausurada los valores fueron 56, 33 y $11 \%$, respectivamente (Tabla 4). La abundancia de plantas dañadas difirió significativamente entre las zonas 

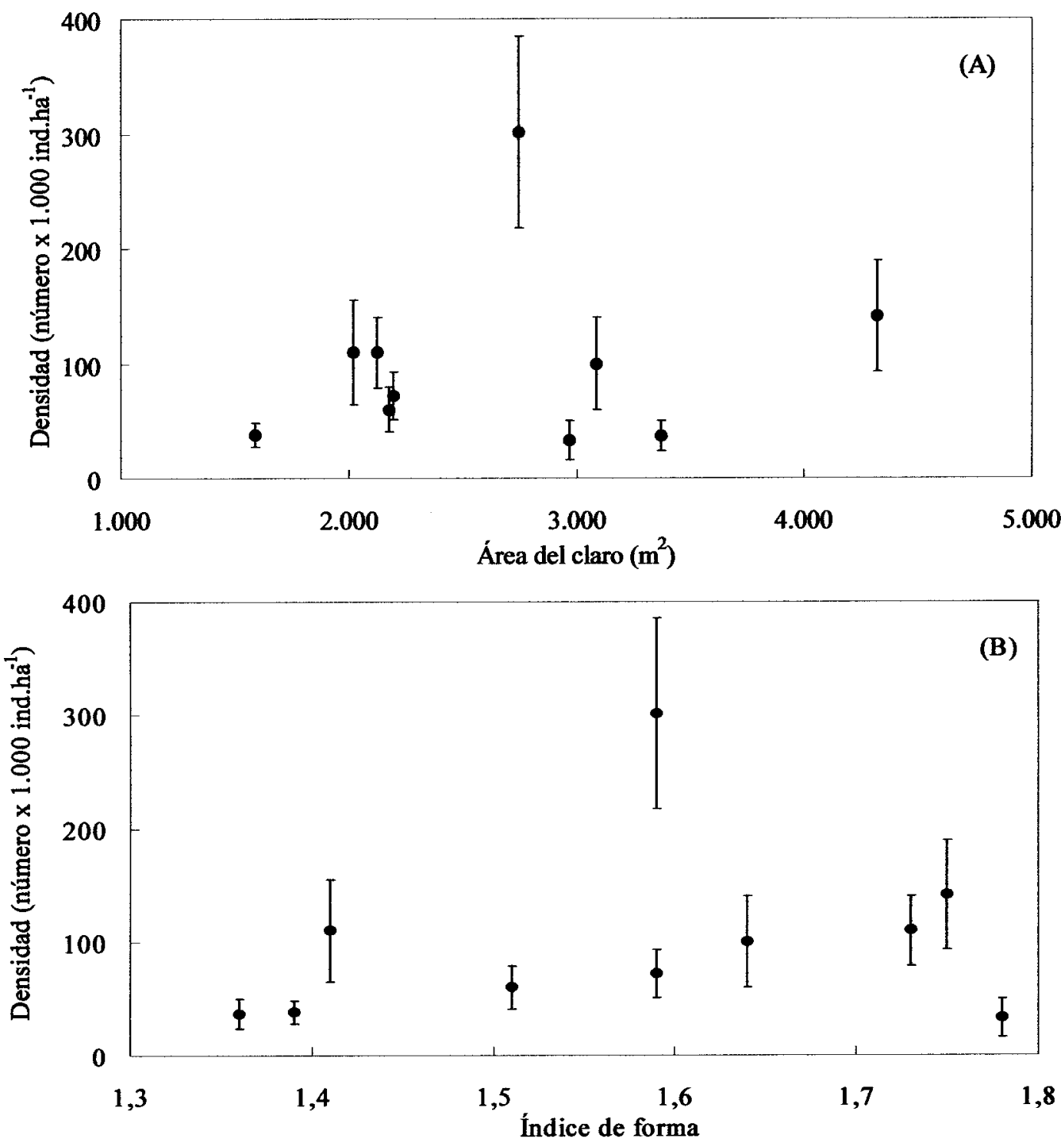

Fig. 4: Densidad de renovales de Nothofagus en función del tamaño expandido (A) y el índice de forma de los claros (B). Las líneas verticales indican el error estándar de la media.

Density of Nothofagus regeneration in relation to expanded area (A) and form index of artificial gaps (B). Vertical lines indicate the standard error of means.

clausurada (21\%) y no clausurada (34\%) (prueba de Kruskal-Wallis; $\left.\mathrm{H}_{2}=5,163 ; \mathrm{P}=0,023\right)$. En la mayoría de los claros se observó una menor proporción de plantas comidas en la zona clausurada con relación a la no clausurada, a excepción del 3 y 4 (Tabla 4). Nothofagus dombeyi exhibió la mayor proporción estadísticamente significativa de plantas sanas tanto en la zona clausurada (83\%) como en la no clausurada (63 $\%$ ) en comparación con $N$. obliqua y $N$. nervosa, mientras que entre estas últimas especies no se observaron diferencias (Tabla 5). Además, $N$. dombeyi exhibió la menor proporción estadísticamente significativa de plantas enfermas tanto en la zona clausurada (4\%) como en la no clausurada $(6 \%)$ en comparación con $N$. obliqua y $N$. nervosa, mientras que entre estas últimas especies no se observaron diferencias (Tabla 5). No se registraron diferencias significativas entre especies en el porcentaje de plantas ramoneadas (Tabla 5).

El $42 \%$ de los renovales se localizaron en las parcelas con un nivel de dominancia del sotobosque bajo $\left(5,9\right.$ ind $\left.0,5 \mathrm{~m}^{-2} ; \mathrm{EE}=0,9\right)$, el 


\section{TABLA 4}

Densidad de los diferentes estados de conservación de los renovales de Nothofagus (S: sano, E: enfermo, C: comido) presentes en las zonas clausurada y no clausurada de cada claro. Se indican la media, el error estándar (EE) y el tamaño de la muestra (n)

Regeneration density of Nothofagus in relation to the status of plants (S: healthy, E: diseased, C: browsed) within the enclosed and open areas of gaps. Mean, standard error (EE), and sample size (n) are showed

\begin{tabular}{|c|c|c|c|c|c|c|c|c|c|c|c|c|}
\hline \multirow{3}{*}{ Claro } & \multicolumn{6}{|c|}{ Zona clausurada } & \multicolumn{6}{|c|}{ Zona no clausurada } \\
\hline & \multirow[b]{2}{*}{$\mathrm{n}$} & \multirow{2}{*}{$\begin{array}{c}S \\
(\%)\end{array}$} & \multirow{2}{*}{$\begin{array}{c}\mathrm{E} \\
(\%)\end{array}$} & \multirow{2}{*}{$\begin{array}{c}\mathrm{C} \\
(\%)\end{array}$} & \multicolumn{2}{|c|}{ Total } & \multirow[b]{2}{*}{$\mathrm{n}$} & \multirow{2}{*}{$\begin{array}{c}S \\
(\%)\end{array}$} & \multirow{2}{*}{$\begin{array}{c}E \\
(\%)\end{array}$} & \multirow{2}{*}{$\begin{array}{c}\mathrm{C} \\
(\%)\end{array}$} & \multicolumn{2}{|c|}{ Total } \\
\hline & & & & & (ind ha-1) & $(\%)$ & & & & & (ind $\mathrm{ha}^{-1}$ ) & $(\%)$ \\
\hline 1 & 61 & 62,3 & 4,9 & 32,8 & 67.778 & 61,3 & 38 & 45,5 & 1,3 & 53,2 & 22.647 & 38,7 \\
\hline 2 & 275 & 77,8 & 1,8 & 20,4 & 203.704 & 69,4 & 121 & 64,4 & 1,7 & 33,9 & 83.448 & 30,6 \\
\hline 3 & 95 & 45,6 & 6,3 & 48,1 & 51.081 & 42,3 & 129 & 56,1 & 10,9 & 32,9 & 103.200 & 57,7 \\
\hline 4 & 28 & 71,5 & 7,1 & 21,4 & 26.667 & 28,3 & 71 & 71,9 & 21,1 & 7,0 & 43.030 & 71,7 \\
\hline 5 & 86 & 58,1 & 12,8 & 29,1 & 71.667 & 46,0 & 101 & 42,6 & 10,9 & 46,5 & 202.000 & 54,0 \\
\hline 6 & 613 & 77,1 & 16,2 & 6,7 & 371.515 & 83,1 & 125 & 50,4 & 15,2 & 34,4 & 156.250 & 16,9 \\
\hline 7 & 42 & 88,1 & 2,4 & 9,5 & 35.000 & 40,0 & 63 & 41,3 & 19,0 & 39,7 & 31.500 & 60,0 \\
\hline 8 & 132 & 86,4 & 3,8 & 9,8 & 105.600 & 51,2 & 126 & 54,8 & 11,1 & 34,1 & 114.545 & 48,8 \\
\hline 9 & 111 & 79,2 & 9,0 & 11,8 & 105.238 & 80,4 & 27 & 48,2 & 11,1 & 40,7 & 21.600 & 19,6 \\
\hline 10 & 168 & 82,2 & 6,5 & 11,3 & 176.842 & 71,5 & 67 & 83,6 & 4,5 & 11,9 & 47.857 & 28,5 \\
\hline Media & & 75,4 & 9,5 & 15,1 & 129.317 & 65,0 & & 56,3 & 10,8 & 32,9 & 66.298 & 35,0 \\
\hline $\mathrm{EE}$ & & 4,3 & 1,4 & 4,2 & 33.274 & 5,9 & & 4,3 & 2,1 & 4,5 & 19.387 & 5,9 \\
\hline
\end{tabular}

TABLA 5

Densidad de renovales (\%) de $N$. dombeyi $(N d), N$. obliqua $(N o)$ y $N$. nervosa $(N n)$ según el estado de conservación. Se indican la media, el error estándar (EE), el tamaño de la muestra (n) y los grupos homogéneos $(\mathrm{GH})$. Letras desiguales indican diferencias entre especies dentro de los estados de conservación (prueba de Kruskal-Wallis; P $\leq 0,05$ )

Regeneration density (\%) of $N$. dombeyi $(N d), N$. obliqua $(N o)$, and $N$. nervosa $(N n)$ in relation to plant condition. Mean, standard error (EE), sample size (n), and homogeneous groups $(\mathrm{GH})$ are showed. Different letters indicate significant differences among species for each condition (Kruskal-Wallis test, $\mathrm{P} \leq 0.05$ )

\begin{tabular}{|c|c|c|c|c|c|c|c|c|c|}
\hline \multirow[t]{2}{*}{ Estadístico } & \multicolumn{3}{|c|}{ Sano } & \multicolumn{3}{|c|}{ Enfermo } & \multicolumn{3}{|c|}{ Dañado } \\
\hline & $N d$ & $\mathrm{No}$ & $\mathrm{Nn}$ & $N d$ & $\mathrm{No}$ & $\mathrm{Nn}$ & $N d$ & $\mathrm{No}$ & $N n$ \\
\hline Media & 71,2 & 49,8 & 56,3 & 3,3 & 18,7 & 19,9 & 25,5 & 31,5 & 23,8 \\
\hline $\mathrm{EE}$ & 2,6 & 3,9 & 4,0 & 0,7 & 2,9 & 3,3 & 2,6 & 3,9 & 3,6 \\
\hline GH & $\mathrm{a}$ & $\mathrm{b}$ & $\mathrm{b}$ & $\mathrm{a}$ & $\mathrm{b}$ & b & $\mathrm{a}$ & $\mathrm{a}$ & a \\
\hline $\mathrm{n}$ & & 170 & & & 95 & & & 110 & \\
\hline $\mathrm{P}$ & & 0,0001 & & & $<0,0001$ & & & 0,0829 & \\
\hline
\end{tabular}

$44 \%$ con un nivel intermedio $\left(6,6\right.$ ind $0,5 \mathrm{~m}^{-2}$; $\mathrm{EE}=1,9)$ y el $14 \%$ con un nivel alto $(2,2$ ind $\left.0,5 \mathrm{~m}^{-2} ; \mathrm{EE}=0,5\right)$. La densidad en las parcelas con niveles de dominancia moderado e intermedio difirió significativamente de la densidad en las parcelas con un nivel alto (prueba de Kruskal-Wallis; H3 = 14,618; $\mathrm{P}<0,0001$ ). En las parcelas con un nivel de dominancia nulo de sotobosque no se observaron renovales y correspondieron a las vías de saca principal, a los caminos y a las áreas de acopio de rollizos. Aproximadamente el $46 \%$ de los renovales de $N$. nervosa y $N$. dombeyi se hallaron en parcelas con un nivel bajo de dominancia del sotobosque, y el $56 \%$ de los renovales de $N$. obliqua se encontraron en parcelas con un nivel intermedio de dominancia del sotobosque (Fig. 5). En las parcelas con un alto nivel de dominancia del sotobosque se presentaron los menores porcentajes de renovales de $N$. nervosa $(20 \%), N$. obliqua $(14 \%)$ у $N$. dombeyi (14\%) (Fig. 5). 


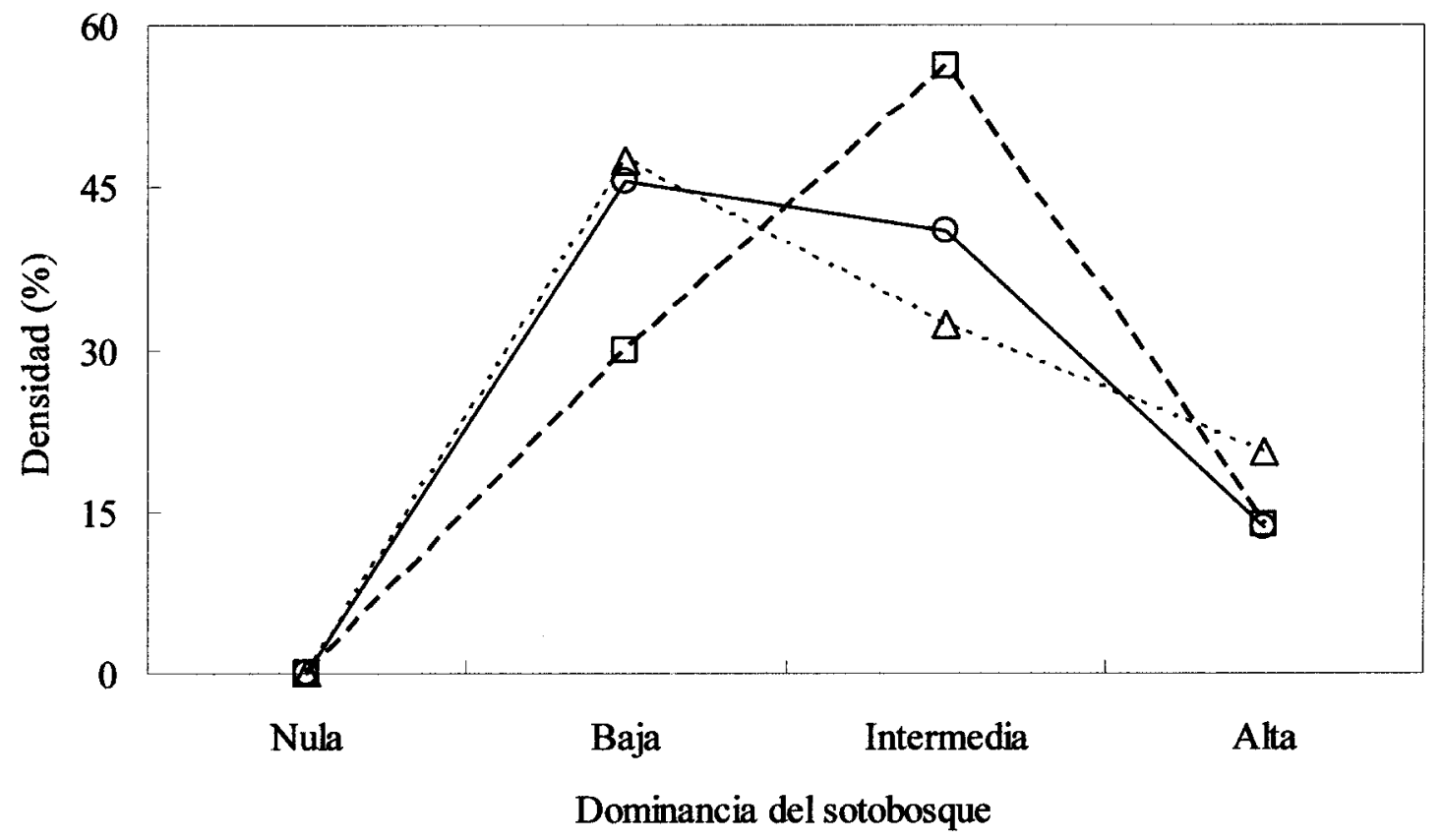

Fig. 5: Densidad (\%) de renovales de $N$. dombeyi (círculos, línea continua), N. obliqua (cuadrados, línea rayada) y $N$. nervosa (triángulos, línea punteada) con relación al nivel de dominancia del sotobosque.

Regeneration density (\%) of $N$. dombeyi (circles, continuous line), $N$. obliqua (squares, dashed line), and $N$. nervosa (triangles, dotted line) in relation to level of understory dominance.

\section{DISCUSIÓN}

Las tres especies de Nothofagus colonizaron los claros artificiales en forma simultánea. La edad media y asimetría de la distribución sensiblemente mayores de $N$. nervosa se debe a la presencia de algunos individuos de entre 8 y 10 años establecidos en las condiciones de baja luminosidad presentes previamente a la implementación de las cortas. La persistencia diferencial de $N$. nervosa como "regeneración avanzada" se explicaría por su mayor tolerancia a la sombra, a juzgar por sus menores tasa de respiración y punto de compensación de luz en comparación con $N$. obliqua y $N$. dombeyi (Read \& Hill 1985). En plantaciones, bajo condiciones de baja luminosidad los juveniles de $N$. nervosa experimentan una mayor sobrevivencia que los de $N$. obliqua, y bajo condiciones de mayor luminosidad esta tendencia se invierte (Grosse 1988a). En bosques adultos, $N$. nervosa presenta una distribución de tamaños asimétrica hacia la izquierda en comparación con $N$. obliqua debido a la mayor persistencia de los individuos de menores tallas debajo de la canopia (Donoso 1993). Además, los renovales de $N$. nervosa son más tolerantes a la sombra que los de $N$. dombeyi a juzgar por su mayor desarrollo y menor mortalidad en condiciones experimentales de sombra (Espinoza 1972, Rosenfeld 1972, Grosse 1988b, Weinberger \& Ramírez 2001).

Los plantines y juveniles pertenecen a la misma cohorte en todas las especies analizadas, a pesar de las marcadas diferencias de tamaño entre estos estados de desarrollo. Los juveniles representan solo el $8 \%$ de los renovales, el $99 \%$ se encuentran en la zona clausurada y exhiben un crecimiento entre 2,6 y 4,8 veces más rápido que los plantines (Dezzotti et al. 2001 $)^{5}$. En poblaciones coetáneas, los factores que promueven la variación de tamaños se relacionan con el mayor tamaño de la semilla, las características genéticas de los individuos, las características físicas del micrositio, la protección contra la herbivoría y la competencia entre individuos (Goldberg 1997, Hutchings 1997). La posición relativa de los

\footnotetext{
${ }^{5}$ DEZZOTTI A, R SBRANCIA, S GOICOECHEA, A RABINO, V FONTANA, M MANZONI, M RODRÍGUEZARIAS, D ROAT, A PARISI, M PEÑALBA \& L MARESCA (2001) Ecología regenerativa de Nothofagus en los bosques templados mixtos de la Patagonia, Argentina. Informe final PI S004. Asentamiento Universitario San Martín de los Andes, Universidad Nacional del Comahue, San Martín de los Andes, Argentina. Manuscrito, 162 pp.
} 
juveniles dentro de la jerarquía de tamaños les confiere mayores expectativas futuras de crecimiento y sobrevivencia y de conformar consecuentemente el rodal adulto.

La comparación de la densidad de renovales actual (97.006 ind ha-1) y la estimada antes de la implementación del plan de manejo (90 ind $\mathrm{ha}^{-1}$ ) indicaría que el tratamiento silvicultural provee las condiciones necesarias para el desarrollo masivo de Nothofagus. Nothofagus dombeyi (68\% del total de plantas) y N. nervosa $(12 \%)$ exhiben el proceso regenerativo más y menos exitoso, respectivamente. La independencia entre la composición específica de renovales y de adultos se asociaría a diferencias entre especies en la producción de semillas viables, la dispersión y germinación de las propágulas y la mortalidad de las plántulas (Harper 1977). Resultados previos sugieren que $N$. dombeyi produce una cantidad significativamente mayor de semillas por individuo (Dezzotti et al. 2001) ${ }^{5}$ con un tamaño y peso significativamente menor en comparación con N. obliqua y N. nervosa (Burschel et al. 1976, Donoso \& Cabello 1978, Ordóñez 1987, Rodríguez 1990, Dezzotti et al. 20015), lo que conduciría a una mayor capacidad de dispersión anemócora. Al igual que las otras especies de Nothofagus de Sudamérica, $N$. dombeyi sería incapaz de formar un banco de semillas persistente en el suelo (Cuevas \& Arroyo 1999, Dezzotti et al. $2001^{5}$ ), por lo que las diferencias de abundancia observadas entre especies no podrían adjudicarse a este proceso.

El efecto protector de la clausura contra la herbivoría se infiere de la mayor densidad de renovales y la menor proporción de plantas comidas en la zona clausurada (129.317 ind $\mathrm{ha}^{-1}, 15 \%$ comidos) con respecto a la zona no clausurada (66.298 ind ha-1 y $33 \%$ comidos), tanto en las áreas centrales como circundantes al alambrado. Solo el $14 \%$ de los renovales se localizaron en parcelas con niveles de dominancia alto del sotobosque que dificultaría el establecimiento y desarrollo de los renovales de Nothofagus. Las parcelas con dominancia nula del sotobosque se localizan en las áreas que estuvieron más afectadas por el proceso de corta, donde se produciría una mayor compactación de la capa superficial de suelo y, consecuentemente, una disminución de su aireación y una pérdida de su capacidad de infiltración de agua (Miller \& Donahue 1995). Aunque la arquitectura de los claros artificiales no influye sobre la densidad actual de los renovales dentro de los rangos estudiados, se espera que en los claros con mayor abundancia las plantas experimenten tasas de mortalidad denso-dependiente e ingresen a la fase de autorraleo más tempranamente.

El reclutamiento natural del bosque de Nothofagus en respuesta a las cortas se considera altamente positivo en el contexto de su uso conservativo. Las opciones silviculturales futuras dependerán de objetivos específicos de manejo de la vegetación. En este sentido, la continua exclusión del ganado de las áreas de regeneración y la manipulación deliberada del ecosistema para favorecer el establecimiento diferencial de $N$. nervosa, la especie que exhibe el menor desempeño y el mayor valor comercial, se deberían considerar en forma prioritaria.

\section{AGRADECIMIENTOS}

Agradecemos la asistencia durante las diferentes instancias del proyecto de L. Chauchard, V. Fontana, S. Goicoechea, J. Puntieri, E. Crivelli, J. Forquera, O. Álvarez y S. Bramardi. Revisores anónimos aportaron valiosas sugerencias al manuscrito. Agradecemos a la Comunidad Mapuche Curruhuinca y la Administración de Parques Nacionales (Parque Nacional Lanín) por permitirnos colectar información en el área de estudio, y a la Fuerza Aérea Argentina por proveernos material fotográfico. The International Foundation of Science (Grant D-2948-1 para AD) y la Universidad Nacional del Comahue (PI S004) financiaron el proyecto.

\section{LITERATURA CITADA}

ALBERDI M (1976) Ecofisiología de especies chilenas del género Nothofagus. Bosque (Chile) 8: 77-84.

APLET G, N JOHNSON, JT OLSON \& VA SAMPLE (1993) Defining sustainable forestry. Island Press, Washington, District of Columbia, USA. 328 pp.

BURSCHEL P, C GALLEGOS, O MARTÍNEZ \& W MOLL (1976) Composición y dinámica regenerativa de un bosque virgen mixto de raulí y coigüe. Bosque (Chile) 1: 55-74.

CABRERA AL (1971) Fitogeografía de la República Argentina. Boletín de la Sociedad Argentina de Botánica 14: 1-42.

CORREA MN (1978) Flora patagónica. Parte III: Gramineae. Colección Científica, Instituto Nacional de Tecnología Agropecuaria, Buenos Aires, Argentina. 563 pp.

CORREA MN (1984) Flora patagónica. Parte IV a: Dicotiledóneas dialipétalas. Colección Científica, Instituto Nacional de Tecnología Agropecuaria, Buenos Aires, Argentina. 559 pp.

CUEVAS JG \& MK ARROYO (1999) Ausencia de banco de semillas persistente en Nothofagus pumilio (Fagaceae) en Tierra del Fuego, Chile. Revista Chilena de Historia Natural 72: 73-82. 
DONOSO C (1993) Bosques templados de Chile y Argentina: variación, estructura y dinámica. Editorial Universitaria, Santiago, Chile. 484 pp.

DONOSO C \& A CABELLO (1978) Antecedentes fenológicos y de germinación de especies leñosas chilenas. Ciencias Forestales (Chile) 1: 31-41.

ESPINOZA M (1972) Alcances sobre las condiciones de luz como factor importante en la regeneración natural del bosque tipo raulí (Nothofagus alpina Poepp. et Endl., Kasser) y coigüe (Nothofagus dombeyi (Mirb.) Oerst.). Tesis de grado, Facultad de Ingeniería Forestal, Universidad Austral de Chile, Valdivia, Chile. 76 pp.

FERRER JA, JA IRIZARRI \& JM MENDÍA (1990) Estudio regional de suelos de la provincia de Neuquén. Consejo Federal de Inversiones, Consejo de Planificación y Acción para el Desarrollo, Neuquén, Argentina. $159 \mathrm{pp}$.

GOLDBERG DE (1997) Competitive ability: definition, contingency, and correlate traits. En: Silvertown JW, M Franco y J Harper (eds) Plant life histories: ecology, phylogeny and evolution: 283-306. Columbia University Press, New York, New York, USA.

GOUDIE A (1993) The human impact on the natural environment. Blackwell Science, Oxford, United Kingdom. $454 \mathrm{pp}$.

GROSSE H (1988a) Crecimiento de plantaciones de raulí y roble bajo dosel en dependencia del grado de luminosidad y fertilización. Ciencia e Investigación Forestal (Chile) 2: 13-30.

GROSSE H (1988b) Desarrollo de plantas de los géneros Fagus y Nothofagus en función de la luminosidad: revisión bibliográfica. Ciencia e Investigación Forestal (Chile) 3: 114-120.

GROSSE H \& I QUIRÓZ (1998) Silvicultura de los bosques de segundo crecimiento de roble, raulí y coigüe en la región centro-sur de Chile. En: Donoso C \& A Lara (eds) Silvicultura de los bosques nativos de Chile: 95-128. Editorial Universitaria, Santiago, Chile.

HARPER J (1977) Population biology of plants. Academic Press, New York, New York, USA. 892 pp.

HILL RS \& G JORDAN (1993) The evolutionary history of Nothofagus (Nothofagaceae). Australian Systematic of Botany 6: 11-126.

HUTCHINGS MJ (1997) The structure of plant populations. En: Crawley MJ (ed) Plant ecology: 325-358. Blackwell Science, Oxford, United Kingdom.

LARA A, C DONOSO, P DONOSO, P NÚÑEZ \& A CAVIERES (1998) Normas de manejo para raleo de renovales del tipo forestal roble-raulí-coigüe. En: Donoso C \& A Lara (eds) Silvicultura de los bosques nativos de Chile: 129-144. Editorial Universitaria, Santiago, Chile.

LARA A, P RUTHERFORD, C MONTORY, D BRAN, A PÉREZ, S CLAYTON, J AYESA, D BARRIOS, M GROSS \& G IGLESIAS (1999) Vegetación de la ecorregión de los bosques valdivianos. Boletín Técnico Fundación Vida Silvestre Argentina 51: 1-29.

LENNON JA, ES MARTIN, RA STEVENS \& DL WIGSTON (1987) Nothofagus nervosa (Phil.) Dim. et Mil. - The correct name for raulí, Chilean southern beech $(N$. procera). Arboricultural Journal 11: 323-332.

MARTÍNEZ-VELÁSQUEZ A (1998) Silvicultura práctica en renovales puros y mixtos, y bosques remanentes originales del tipo forestal roble-raulí-coigüe. En: Donoso C \& A Lara (eds) Silvicultura de los bosques nativos de Chile: 145-175. Editorial Universitaria, Santiago, Chile.
MILLER RW \& RL DONAHUE (1995) Soils in our environment. Prentice-Hall Inc., Englewood Cliffs, New Jersey, USA. 650 pp.

MORELLO J \& S MATTEUCCI (1999) Biodiversidad y fragmentación de los bosques en la Argentina. En: Matteucci S, O Solbrig, J Morello \& G Halffter (eds) Biodiversidad y uso de la tierra: conceptos y ejemplos de Latinoamérica: 463-498. Colección CEA 24, Editorial Universitaria de Buenos Aires, Buenos Aires, Argentina.

ORDÓÑEZ A (1987) Germinación de tres especies de Nothofagus siempreverdes (coigües) y variabilidad en la germinación de procedencias de coigüe común (Nothofagus dombeyi, (Mirb.) Oerst.). Tesis de grado, Facultad de Ciencias Forestales, Universidad Austral de Chile, Valdivia, Chile. 134 pp.

POOLE AL (1987) Southern beeches. New Zealand Department of Scientific and Industrial Research Information Series 162. Science Information Publishing Centre, Wellington, New Zealand. 148 pp.

RAISON RJ, DW FLINN \& AG BROWN (2001) Application of criteria and indicators to support sustainable forest management: some key issues. En: Raison RJ, AG Brown \& DW Flinn (eds) Criteria and indicators for sustainable forest management: 5-18. International Union of Forestry Research Organizations Research Series 7, CABI Publishing, Wallingford, United Kingdom.

RAMÍREZ C, C SAN MARTÍN, A OYARZÚN \& H FIGUEROA (1997) Morpho-ecological study of the South American species of the genus Nothofagus. Plant Ecology 130: 101-109.

READ J \& RS HILL (1985) Photosynthetic responses to light of Australian and Chilean species of Nothofagus and their relevance to the rain forest dynamics. New Phytologist 101: 731-743.

RODRÍGUEZ G (1990) Propagación de Nothofagus chilenos por medio de semillas. Agro-Ciencia (Chile) 6: $119-122$

ROSENFELD JM (1972) Desarrollo de la regeneración de raulí (Nothofagus alpina) y coigüe (Nothofagus dombeyi) bajo diferentes grados de luminosidad. Tesis de grado, Facultad de Ingeniería Forestal, Universidad Austral de Chile, Valdivia, Chile. 63 pp.

RUNKLE JR (1992) Guidelines and sample protocol for sampling forest gaps. United States Department of Agriculture, Forest Service General Technical Report PNW-GTR-283, Portland, Oregon, USA. 44 pp.

SMITH DM, BC LARSON, MJ KELTY \& PM ASHTON (1997) The practice of silviculture: applied forest ecology. John Wiley \& Sons, New York, New York, USA. $537 \mathrm{pp}$

SOKAL R \& F ROHLF (1995) Biometry. WH Freeman, New York., U.S.A. 887 pp.

VEBLEN TT (1989) Nothofagus regeneration in treefall gaps in northern Patagonia. Canadian Journal of Forest Research 19: 365-371.

VEBLEN TT, C DONOSO, FM SCHLEGEL \& R ESCOBAR (1981) Forest dynamics in south-central Chile. Journal of Biogeography 8: 211-247.

WAGNER RG \& JC ZASADA (1991) Integrating plant autoecology and silvicultural activities to prevent forest vegetation management problems. Forestry Chronicle 67: 506-513.

WEINBERGER P \& C RAMÍREZ (2001) Microclima y regeneración natural de raulí, roble y coigüe (Nothofagus alpina, N. obliqua y N. dombeyi). Bosque (Chile) 22: 11-26. 